\title{
Volume Flexibility in Production Model with Cubic Demand Rate and Weibull Deterioration with partial backlogging.
}

\author{
Dr. Ravish Kumar $\operatorname{Yadav}^{1}$, Ms. Pratibha Yadav ${ }^{2}$
}

\begin{abstract}
In the present paper a volume flexible production inventory model is developed for deteriorating items with time dependent demand rate. The demand rate is taken as cubic function of time and production rate is decision variable. Production cost becomes a function of production rate. Unit production cost is depending upon material cost, Labor cost and tool or die cost. The deteriorating of unit in an inventory system is taken to weibull distribution. Shortage with partially backlogged are allowed a very natural phenomenon in inventory model.
\end{abstract}

\section{Introduction}

Most of the physical goods undergo decay of deteriorate over time. Commodities such as fruits, vegetables, foodstuffs etc. suffer from depletion by direct spoilage while kept in store. Highly volatile liquids such as gasoline, alcohol, turpentine etc. Undergo physical depletion over time through the process of evaporation. Electronic goods, radioactive substances, photographic film, grain etc. deteriorates through a gradual loss of potential or utility with the passage of time. Thus decay or deterioration of physical goods in stock is a very realistic feature and inventory modelers felt the need to take this factor into consideration.

Some modelers have taken constant deterioration rate in their inventory models. Giri and Chaudhuri (1998), Chang and Dye (1999), Chung (2000), Papachristos and Skouri (2000), Kumar and Sharma (2000), Aggarwal and Jain (2001), Khanra and Chaudhuri (2003), Sana et al (2004), Teng, Chang and Goyal (2005) have used constant deterioration their inventory models. But the concept of constant deterioration is not realistic in real life situations. Therefore in our inventory model, we have taken variable deterioration rate.

Schweitzer and Seidmann (1991) were the first one to have broken the ice in this direction when they enlightened the concept of flexibility in the machine production rate and discussed optimization of processing rate for a FMS (flexible manufacturing system). Obviously, the machine production rate. Silver (1990)discussed the effect of showing down production in the context of a manufacturing equipment of a family of items, assuming a common cycle for all the items. Ramesh and jaya Kumar (1991) used the shape of the average unit cost curve to measure volume flexibility. Flat average unit cost curve indicates a volume flexible production system. Average unit cost to increase.. Gallego (1993) extended the model of Silver (1990) by removing the stipulation of a common cycle for all the items. Khouja and Mehrez (1994) and Khouja (1995) and Khouja (1995) extended the EPLS (Economic lot size production) model to an imperfect production process with a flexible production rate.

Sana and Chaudhuri (2003) considered volume flexibility for deteriorating item with an inventory level dependent demand rate. Sana and Chaudhuri (2004) developed inventory model with volume flexible production for deteriorating completely backlogged, Sana (2004) extended EPLS model in which the rate of production depends upon the technology of manufacturing system, capital investment for MRP and powerful with time dependent rate. Khouja (2005) extended the EPLS

1, Associate Professor, Hindu College, Moradabad, Email:drravishyadav@yahoo.com

${ }^{2}$, Research Scholar, Hindu College, Moradabad model to allow revisions to the demand forecast and multiple production rates for the linear penalty case. Husseini and Brein (2006) discussed a method to enhance volume flexibility in JIT production control. Sana et al. (2006) discussed the effect of slowing down production in the context of a manufacturing equipment of a family of items. Sana et al. (2007) and Sana and Chaudhuri (2007) extended the EPLS model which accounts for a production system producing items of perfect as well as imperfect quality with volume FMS. Most of the references cited above have considered a deteriorating stock.

Further more, when the shortages occur, same customers are willing to wait for backorder and others would turn to buy from other sellers. Many researchers such as Park(1982), Hollier and mak (1983) and Wee (1995) considered the constant partial backlogging rates during the shortage period in their inventory models. In some inventory systems, such as fashionable commodities the length of the waiting time for the next replenishment would determine whether the backlogging would be accepted or not. Therefore, the backlogging rate is variable and dependent on the length of the waiting time for the next replenishment. Abad (1996) investigated an EOQ model allowing shortage and partial backlogging. 
Some researchers, Balkhi and Benkherouf (1996), Chu and Chung (2004) and Giri and Yun (2005) developed inventory model with constant demand rate. But the concept of constant demand rate is not so realistic. Therefore we have taken variable demand in our model.

In this model, we have taken deterioration rate as two parameter weibull distribution and demand rate cubic increasing of time and production rate is variable. Shortages are allowed and partially backlogged and backlogging rate is dependent on waiting time for next replenishment.

\section{Assumptions And Notations}

The production inventory model for deteriorating items is developed on the basis of the following assumptions and notationss.

(i) $\quad \mathrm{I}(\mathrm{t})$ is the inventory level at any time $t \geq 0$.

(ii) The demand rate $\mathrm{R}(\mathrm{t})=\mathrm{a}+\mathrm{bt}+\mathrm{ct}^{2}+\mathrm{dt}^{3}$, where a, b, c and d are the positive constants and $0<\mathrm{b}<<$ 1.

(iii) The production rate $\mathrm{K}$ is variable.

(iv) A fraction $\theta(t)=\alpha \beta t^{\beta-1}, 0<\alpha<<1, t>0, \beta \geq 1$ of the on hand inventory deteriorates per unit time.

(v) The deteriorating units can be neither replaced nor repaired during the cycle time.

(vi) The lead-time is zero.

(vii) $\mathrm{C}^{\mathrm{I}}, \mathrm{C}_{\mathrm{H}}, \mathrm{C}_{\mathrm{S}}, \mathrm{C}_{\mathrm{D}}, \mathrm{C}_{\mathrm{L}}$ denote the set up cost for each replenishment, inventory carrying cost per unit time, shortage cost for backlogged items, deterioration cost per unit, the unit cost of last sales respectively. All of the cost parameters are positive constants.

(viii) Shortages are allowed and backlogging rate is taken as $\frac{1}{1+\delta(T-t)}$, where $\delta$ is the backlogging parameter and $0<\delta<1$.

(ix) Unit production cost $\left[\eta(K)=R+\frac{G}{K}+H K\right]$ is depends upon the production rate, where $\mathrm{R}, \mathrm{G}$ and $\mathrm{H}$ are the material cost, labour cost and tool or dye cost respectively.

(x) $\mathrm{T}$ is the time horizon.

(xi) A single item is considered over the prescribed period of time

\section{Mathematical Model And Analysis}

Initially the inventory level is zero. The production starts at time $t=0$ and after $t_{1}$, units of time, it reaches to maximum inventory level. After this production stopped and at time $t=t_{2}$, the inventory level becomes zero. At this time shortages starts developing at time $t=t_{3}$, it reaches to maximum shortages level. At this fresh production starts to clear the backlog by the time $t=T$. Our aim is to find optimum values of $t_{1}, t_{2}$, $\mathrm{t}_{3}$, $\mathrm{T}$ that minimize. The total average cost $\mathrm{C}$ over time horizon $(0, \mathrm{~T})$.

Let $\mathrm{I}(\mathrm{t})$ be the inventory level at any time $\mathrm{t}, 0 \leq t \leq T$.

The governing differential equations of an inventory systems in the interval $[0, \mathrm{~T})$ are

$$
\begin{array}{cc}
\frac{d I(t)}{d t}+\theta(t) I(t)=K-R(t), & 0 \leq t \leq t_{1} \\
\frac{d I(t)}{d t}+\theta(t) I(t)=-R(t), & t_{1} \leq t \leq t_{2} \\
\frac{d I(t)}{d t} \quad=-\frac{1}{1+\delta(T-t)} R(t), & t_{2} \leq t \leq t_{3} \\
\frac{d I(t)}{d t} \quad=K-R(t), & t_{3} \leq t \leq T
\end{array}
$$

with the condition $I(0)=I\left(t_{2}\right)=I(T)=0$, using the value of $\mathrm{R}(\mathrm{t})$ and $\theta(\mathrm{t})$ the equation (1), (2), (3) and (4) gives

$$
\begin{aligned}
& \frac{d I(t)}{d t}+\alpha \beta t^{\beta-1} I(t)=K-\left(a+b t+c t^{2}+d t^{3}\right), \quad 0 \leq t \leq t_{1} \\
& \frac{d I(t)}{d t}+\alpha \beta t^{\beta-1} I(t)=-\left(a+b t+c t^{2}+d t^{3}\right), t_{1} \leq t \leq t_{2} \\
& \frac{d I(t)}{d t}=-\frac{1}{1+\delta(T-t)}\left(a+b t+c t^{2}+d t^{3}\right), \quad t_{2} \leq t \leq t_{3} \\
& \frac{d I(t)}{d t}=K-\left(a+b t+c t^{2}+d t^{3}\right), t_{3} \leq t \leq T
\end{aligned}
$$

Solution of equation (5), (6), (7) and (8) are:

$$
\begin{aligned}
& I(t)=\left[K\left(t-\frac{\alpha \beta t^{\beta+1}}{\beta+1}\right)-\left(a t+\frac{b t^{2}}{2}+\frac{c t^{3}}{3}+\frac{d t^{4}}{4}-\frac{a \alpha \beta t^{\beta+1}}{\beta+1}-\frac{b \alpha \beta t^{\beta+2}}{2(\beta+2)}-\frac{c \alpha \beta t^{\beta+3}}{3(\beta+3)}-\quad 0 \quad \frac{d \alpha \beta t^{\beta+4}}{4(\beta+4)}\right)\right] e^{-\alpha t^{\beta}} \\
& I(t)=\left[a\left(t_{2}-t\right)+\frac{b}{2}\left(t_{2}^{2}-t^{2}\right)+\frac{c}{3}\left(t_{2}^{3}-t^{3}\right)+\frac{d}{4}\left(t_{2}^{4}-t^{4}\right)+\frac{a \alpha \beta t^{\beta+1}}{\beta+1}+\quad \frac{\ldots .(9)}{\beta+2}+\frac{b \alpha \beta t^{\beta+2}}{\beta+2}+\frac{c \alpha \beta t^{\beta+3}}{\beta+3}+\right. \\
& \left.\frac{a \alpha t_{2}^{\beta+1}}{\beta+1}+\frac{b \alpha t_{2}^{\beta+2}}{\beta+2}+\frac{c \alpha t_{2}^{\beta+3}}{\beta+3}+\frac{d \alpha t_{2}^{\beta+4}}{\beta+4}-a \alpha t_{2} t^{\beta}-\quad \frac{b \alpha t_{2}^{2} t^{\beta}}{2}-\frac{c \alpha t_{2}^{3} t^{\beta}}{3}-\frac{d \alpha t_{2}^{4} t^{\beta}}{4}\right] e^{-\alpha t^{\beta}}
\end{aligned}
$$


$t_{1} \leq t \leq t_{2}$

$$
\begin{aligned}
& I(t)=\frac{1}{6 \delta^{4}}\left[6\left\{b \delta^{3}+c \delta^{2}(1+\delta T)+d \delta(1+\delta T)^{2}\right\}\left(t-t_{2}\right)+3\left\{c \delta^{3}+\quad d \delta^{4}(1+\delta T)\right\}\{[1+\delta(T-\right. \\
& \left.t)]^{2}-\left[1+\delta\left(T-t_{2}\right)\right]^{2}\right\}+2 d\left\{[1+\delta(T-t)]^{3}-\left[1+\delta\left(T-t_{2}\right)\right]^{3}\right\}+6\left\{a \delta^{3}+b \delta^{2}(1+\delta T)+\right. \\
& \left.\left.c \delta(1+\delta T)^{2}+\quad d(1+\delta T)^{3}\right\} \log \left\{\frac{1+\delta(T-t)}{1+\delta\left(T-t_{2}\right)}\right\}\right] \quad t_{2} \leq t \leq t_{3} \\
& \begin{array}{r}
I(t)=K(t-T)+a(T-t)+\frac{b}{2}\left(T^{2}-t^{2}\right)+\frac{c}{3}\left(T^{3}-t^{3}\right)+\frac{d}{4}\left(T^{4}-t^{4}\right) \\
t_{3} \leq t \leq T
\end{array}
\end{aligned}
$$

Total number of unit holding is given by

$$
\begin{gathered}
I_{H}=\int_{0}^{t_{1}} I(t) d t+\int_{t_{1}}^{t_{2}} I(t) d t \\
I_{H}=K\left(\frac{t_{1}^{2}}{2}-\frac{\alpha \beta t_{1}^{\beta+2}}{(\beta+1)(\beta+2)}\right)+\frac{a t_{2}^{2}}{2}+\frac{b t_{2}^{3}}{3}+\frac{c t_{2}^{4}}{4}+\frac{d t_{2}^{5}}{5}+\frac{a \alpha \beta t_{2}^{\beta+2}}{(\beta+1)(\beta+2)} \\
+\frac{b \alpha \beta t_{2}^{\beta+3}}{(\beta+1)(\beta+3)}+\frac{c \alpha \beta t_{2}^{\beta+4}}{(\beta+1)(\beta+4)}+\frac{d \alpha \beta t_{2}^{\beta+5}}{(\beta+1)(\beta+5)}+t_{1} t_{2}\left(a+\frac{b t_{2}}{2}+\frac{c t_{2}^{2}}{3}+\frac{d t_{2}^{3}}{4}\right) \\
\left(\frac{\alpha t_{1}^{\beta}}{\beta+1}-1\right)-\alpha t_{1} t_{2}^{\beta+1}\left(\frac{a}{\beta+1}+\frac{b t_{2}}{\beta+2}+\frac{c t_{2}^{2}}{\beta+3}+\frac{d t_{2}^{3}}{\beta+4}\right)
\end{gathered}
$$

Total amount of deteriorated units is given by

$$
\begin{aligned}
I_{D}=\int_{0}^{t_{1}} \alpha \beta t^{\beta-1} I(t) d t+\int_{t_{1}}^{t_{2}} \alpha \beta t^{\beta-1} I(t) d t \\
I_{D}=\frac{k \alpha \beta t_{1}^{\beta+1}}{\beta+1}-\alpha t_{1}^{\beta} t_{2}\left(a+\frac{b t_{2}}{2}+\frac{c t_{2}^{2}}{3}+\frac{d t_{2}^{4}}{4}\right)+\frac{a \alpha t_{2}^{\beta+1}}{\beta+1}+\frac{b \alpha t_{2}^{\beta+2}}{\beta+2}+\frac{c \alpha t_{2}^{\beta+3}}{\beta+3}+\quad \frac{d \alpha t_{2}^{\beta+4}}{\beta+4}
\end{aligned}
$$

Total number of shortage unit is given by

$$
I_{S}=-\int_{t_{2}}^{t_{3}} I(t) d t-\int_{t_{3}}^{T} I(t) d t
$$

$$
\begin{aligned}
& = \\
& \frac{1}{6 \delta^{4}}\left[3\left\{b \delta^{3}+c \delta^{2}(1+\delta T)+d \delta \quad(1+\delta T)^{2}\right\}\left(t_{3}-t_{2}\right)^{2}-\right. \\
& 3\left\{c \delta^{3}+\quad d \delta^{4}(1+\delta T)\right\}\left\{\frac{\left[1+\delta\left(T-t_{3}\right)\right]^{3}-\left[1+\delta\left(T-t_{2}\right)\right]^{3}}{3 \delta}+\left[1+\delta\left(T-t_{2}\right)\right]^{2}\left(t_{3}-t_{2}\right)\right\}- \\
& \quad 2 d\left\{\frac{\left[1+\delta\left(T-t_{3}\right)\right]^{4}-\left[1+\delta\left(T-t_{2}\right)\right]^{4}}{4}+\left[1+\delta\left(T-t_{2}\right)\right]^{3}\left(t_{3}-t_{2}\right)\right\} 6\left\{a \delta^{3}+\quad b \delta^{2}(1+\delta T)+c \delta \quad(1+\delta T)^{2}+\right. \\
& \left.\left.\quad d(1+\delta T)^{3}\right\} \log \left\{\left(t_{3}-\frac{1+\delta T}{\delta}\right) \log \left(\frac{1+\delta\left(T-t_{3}\right)}{1+\delta\left(T-t_{2}\right)}\right)-\left(t_{3}-t_{2}\right)\right\}\right]- \\
& \quad\left[T^{2}\left(\frac{a}{2}+\frac{b T}{3}+\frac{c T^{2}}{4}+\frac{d T^{2}}{5}-\frac{k}{2}\right)-T t_{3}\left(a+\frac{b T}{2}+\frac{c T^{2}}{3}+\frac{d T^{3}}{4}-K\right)\right. \\
& \left.\quad+\frac{t_{3}^{2}}{2}\left(a+\frac{b t_{3}}{3}+\frac{c t_{3}^{2}}{6}+\frac{d t_{3}^{3}}{10}-K\right)\right]
\end{aligned}
$$

Total number of lost sale is given by

$I_{L}=\int_{t_{3}}^{T}\left[1-\frac{1}{1+\delta(T-t)}\right]\left(a+b t+c t^{2}+d t^{3}\right) \mathrm{dt}$

$I_{L}=a\left(T-t_{3}\right)+\frac{b}{2}\left(T^{2}-t_{3}^{2}\right)+\frac{c}{3}\left(T^{3}-t_{3}^{3}\right)+\frac{d}{4}\left(T^{4}-t_{3}^{4}\right)+\frac{1}{6 \delta^{4}}\left[6\left\{b \delta^{3}+c \delta^{2}(1+\delta T)+d \delta \quad(1+\right.\right.$ $\left.\delta T)^{2}\right\}\left(T-t_{3}\right)+3\left\{c \delta^{3}+d \delta^{4}(1+\delta T) \quad\right\}\left\{1-\quad\left[1+\delta\left(T-t_{3}\right)\right]^{2}\right\}+2 d\{1-$

$\left.\left.\left[1+\delta\left(T-t_{3}\right)\right]^{3}\right\}-6\left\{a \delta^{3}+b \delta^{2}(1+\delta T)+\quad c \delta(1+\delta T)^{2}+d(1+\delta T)^{3}\right\} \log \left[1+\delta\left(T-t_{3}\right)\right]\right]$

Form equations (9) we have

$$
\begin{aligned}
& I\left(t_{1}\right)=K\left(t_{1}-\frac{\alpha \beta t_{1}^{\beta+1}}{\beta+1}\right) \\
& \quad-\left(a t_{1}+\frac{b t_{1}^{2}}{2}+\frac{c t_{1}^{3}}{3}+\frac{d t_{1}^{4}}{4}-\frac{a \alpha \beta t_{1}^{\beta+1}}{\beta+1}-\frac{b \alpha \beta t_{1}^{\beta+2}}{2(\beta+2)}-\frac{c \alpha \beta t_{1}^{\beta+3}}{3(\beta+3)}-\frac{d \alpha \beta t_{1}^{\beta+4}}{4(\beta+4)}\right)
\end{aligned}
$$

Form equations (10) have

$$
I\left(t_{1}\right)=\left[a\left(t_{2}-t_{1}\right)+\frac{b}{2}\left(t_{2}^{2}-t_{1}^{2}\right)+\frac{c}{3}\left(t_{2}^{3}-t_{1}^{3}\right)+\frac{d}{4}\left(t_{2}^{4}-t_{1}^{4}\right)+\frac{\alpha \alpha \beta t_{1}^{\beta+1}}{\beta+1}\right.
$$




$$
\begin{aligned}
& \quad+\frac{b \alpha \beta t_{1}^{\beta+2}}{2(\beta+2)}+\frac{c \alpha \beta t_{1}^{\beta+3}}{3(\beta+3)}+\frac{d \alpha \beta t_{1}^{\beta+4}}{4(\beta+4)}+\frac{a \alpha t_{2}^{\beta+1}}{\beta+1}+\frac{b \alpha t_{2}^{\beta+2}}{\beta+2}+\frac{c \alpha t_{2}^{\beta+3}}{\beta+3}+\frac{d \alpha t_{2}^{\beta+4}}{\beta+4}-\quad a \alpha t_{2} t_{1}^{\beta}-\frac{b \alpha t_{2}^{2} t_{1}^{\beta}}{2}-\frac{c \alpha t_{2}^{3} t_{1}^{\beta}}{3}- \\
& \left.\frac{d \alpha t_{2}^{4} t_{1}^{\beta}}{4}\right]
\end{aligned}
$$

By equations (17) and (18), we get

$$
K\left(t_{1}-\frac{\alpha \beta t_{1}^{\beta+1}}{\beta+1}\right)
$$

$$
\begin{aligned}
& =\left(a t_{2}+\frac{b t_{2}^{2}}{2}+\frac{c t_{2}^{3}}{3}+\frac{d t_{2}^{4}}{4}-\frac{a \alpha t_{2}^{\beta+1}}{\beta+1}-\frac{b \alpha t_{2}^{\beta+2}}{\beta+2}-\frac{c \alpha \beta t_{2}^{\beta+3}}{\beta+3}-\frac{d \alpha \beta t_{2}^{\beta+4}}{\beta+4}-a \alpha t_{2} t_{1}^{\beta}-\frac{b \alpha t_{2}^{2} t_{1}^{\beta}}{2}\right. \\
& \left.-\frac{c \alpha t_{2}^{\beta} t_{1}^{\beta}}{2}-\frac{d \alpha t_{2}^{3} t_{1}^{\beta}}{3}\right)
\end{aligned}
$$

Now we consider as

$$
t_{2}=f\left(t_{1}\right)
$$

From equation ( 17$)$

$I\left(t_{3}\right)=\frac{1}{6 \delta^{4}}\left[6\left\{b \delta^{3}+c \delta^{2}(1+\delta T)+d \delta(1+\delta T)^{2}\right\}\left(t_{3}-t_{2}\right)+3\left\{c \delta^{3}+\quad d \delta^{4}(1+\delta T) \quad\right\}\{[1+\delta(T-\right.$ $\left.\left.\left.t_{3}\right)\right]^{2}-\left[1+\delta\left(T-t_{2}\right)\right]^{2}\right\}+2 d\left\{\left[1+\delta\left(T-t_{3}\right)\right]^{3}-\left[1+\delta\left(T-t_{2}\right)\right]^{3}\right\}+6\left\{a \delta^{3}+b \delta^{2}(1+\delta T)+\right.$ $\left.\left.c \delta(1+\delta T)^{2}+\quad d(1+\delta T)^{3}\right\} \log \left\{\frac{1+\delta\left(T-t_{3}\right)}{1+\delta\left(T-t_{2}\right)}\right\}\right]$

From equation (12), we have

$$
I\left(t_{3}\right)=K\left(t_{3}-T\right)+a\left(T-t_{3}\right)+\frac{b}{2}\left(T^{2}-t_{3}^{2}\right)+\frac{c}{3}\left(T^{3}-t_{3}^{3}\right)+\frac{d}{4}\left(T^{4}-t_{3}^{4}\right)
$$

On comparing equations (20) and (21), we get

$$
\begin{aligned}
& \frac{1}{6 \delta^{4}}\left[6\left\{b \delta^{3}+c \delta^{2}(1+\delta T)+d \delta(1+\delta T)^{2}\right\}\left(t_{3}-t_{2}\right)+3\left\{c \delta^{3}+d \delta^{4}(1+\delta T)\right\}\left\{\left[1+\delta\left(T-t_{3}\right)\right]^{2}-\right.\right. \\
& \left.\left[1+\delta\left(T-t_{2}\right)\right]^{2}\right\}+2 d\left\{\left[1+\delta\left(T-t_{3}\right)\right]^{3}-\left[1+\delta\left(T-t_{2}\right)\right]^{3}\right\}+6\left\{a \delta^{3}+b \delta^{2}(1+\delta T)+c \delta \quad(1+\delta T)^{2}+\right. \\
& \left.\left.d(1+\delta T)^{3}\right\} \log \left\{\frac{1+\delta\left(T-t_{3}\right)}{1+\delta\left(T-t_{2}\right)}\right\}\right]=K\left(t_{3}-T\right)+a\left(T-t_{3}\right)+\frac{b}{2}\left(T^{2}-t_{3}^{2}\right)+\frac{c}{3}\left(T^{3}-t_{3}^{3}\right)+\frac{d}{4}\left(T^{4}-t_{3}^{4}\right)
\end{aligned}
$$

Now we consider as

$$
t_{3}=g\left(T, t_{1}\right)
$$

Unit production cost $\quad \eta(K)=R+\frac{G}{K}+H K$

Production cost (P.C.) $=\eta(K) K\left[t_{1}+\left(T-t_{3}\right)\right]$

$$
\Rightarrow \quad P . C .=\left(R+\frac{G}{H}+H K\right) K\left[t_{1}+\left(T-t_{3}\right)\right]
$$

Hence the total average cost of the per unit time is given by

$$
\begin{aligned}
& C=\frac{1}{T}\left[C^{I}+P . C .+C_{H} I_{H}+C_{D} I_{D}+C_{S} I_{S}+C_{L} I_{L}\right] \\
& C=\frac{1}{T}\left[C^{I}+\left(R+\frac{G}{K}+H K\right) K\left[t_{1}+\left\{T-g\left(T_{3} t_{1}\right)\right\}\right]\right] \\
& +C_{H}\left\{K\left(\frac{t_{1}^{2}}{2}-\frac{\alpha \beta t_{1}^{\beta+2}}{(\beta+1)(\beta+2)}\right)+\frac{a\left(f\left(t_{1}\right)\right)^{2}}{2}+\frac{b\left(f\left(t_{1}\right)\right)^{3}}{3}+\frac{c\left(f\left(t_{1}\right)\right)^{3}}{4}+\frac{d\left(f\left(t_{1}\right)\right)^{5}}{5}+\quad \frac{a \alpha \beta\left(f\left(t_{1}\right)\right)^{\beta+2}}{(\beta+1)(\beta+2)}+\frac{b \alpha \beta\left(f\left(t_{1}\right)\right)^{\beta+3}}{(\beta+1)(\beta+3)}+\right. \\
& \frac{c \alpha \beta\left(f\left(t_{1}\right)\right)^{\beta+4}}{(\beta+1)(\beta+4)}+\frac{d \alpha \beta\left(f\left(t_{1}\right)\right)^{\beta+5}}{(\beta+1)(\beta+5)}+\quad t_{1} f\left(t_{1}\right)\left(a+\frac{b f\left(t_{1}\right)}{2}+\frac{c\left(f\left(t_{1}\right)\right)^{2}}{3}+\frac{d\left(f\left(t_{1}\right)\right)^{3}}{4}\right)\left(\frac{\alpha t_{1}^{\beta}}{\beta+1}-1\right)- \\
& \quad \alpha t_{1}\left(f\left(t_{1}\right)\right)^{\beta+1}\left(\frac{a}{\beta+1}+\frac{b f\left(t_{1}\right)}{\beta+2}+\frac{c\left(f\left(t_{1}\right)\right)^{2}}{\beta+3}+\frac{d\left(f\left(t_{1}\right)\right)^{3}}{\beta+4}\right)+C_{D}\left\{\frac{k \alpha \beta t_{1}^{\beta+1}}{\beta+1}-\alpha t_{1}^{\beta} f\left(t_{1}\right)\left(a+\frac{b f\left(t_{1}\right)}{2}+\frac{c\left(f\left(t_{1}\right)\right)^{2}}{3}+\right.\right. \\
& \left.\left.\frac{d\left(f\left(t_{1}\right)\right)^{3}}{4}\right)+\frac{a \alpha\left(f\left(t_{1}\right) \beta^{\beta+1}\right.}{\beta+1}+\frac{b \alpha\left(f\left(t_{1}\right) \beta^{\beta+2}\right.}{\beta+2}+\frac{c \alpha\left(f\left(t_{1}\right)\right)^{\beta+3}}{\beta+3}+\quad \frac{d \alpha\left(f\left(t_{1}\right) \beta^{\beta+4}\right.}{\beta+4}\right\}+\frac{C_{S}}{6 \delta^{4}}\left[3 \left\{b \delta^{3}+c \delta^{2}(1+\delta T)+\right.\right. \\
& \left.d \delta \quad(1+\delta T)^{2}\right\}\left(g\left(T, t_{1}\right)-f\left(t_{1}\right)\right)^{2} 3\left\{c \delta^{3}+\quad d \delta^{4}(1+\delta T) \quad\right\}\left\{\frac{\left[1+\delta\left(T-g\left(T, t_{1}\right)\right)\right]^{3}-\left[1+\delta\left(T-f\left(t_{1}\right)\right)\right]^{3}}{3 \delta}+\right. \\
& \left.\left[1+\delta\left(T-f\left(t_{1}\right)\right)\right]^{2}\left(g\left(T, t_{1}\right)-f\left(t_{1}\right)\right)\right\}-\quad 2 d\left\{\frac{\left[1+\delta\left(T-g\left(T, t_{1}\right)\right)\right]^{4}-\left[1+\delta\left(T-f\left(t_{1}\right)\right)\right]^{4}}{4}+[1+\delta(T-\right. \\
& \left.\left.\left.f\left(t_{1}\right)\right)\right]^{3}\left(g\left(T, t_{1}\right)-f\left(t_{1}\right)\right)\right\} 6\left\{a \delta^{3}+\quad b \delta^{2}(1+\delta T)+c \delta \quad(1+\delta T)^{2}+\quad d(1+\delta T)^{3}\right\} \log \left\{\left(g\left(T, t_{1}\right)-\right.\right. \\
& \left.\left.\left.\frac{1+\delta T}{\delta}\right) \log \left(\frac{1+\delta\left(T-g\left(T, t_{1}\right)\right)}{1+\delta\left(T-f\left(t_{1}\right)\right)}\right)-\left(g\left(T, t_{1}\right)-f\left(t_{1}\right)\right)\right\}\right]-\left[T^{2}\left(\frac{a}{2}+\frac{b T}{3}+\frac{c T^{2}}{4}+\frac{d T^{2}}{5}-\frac{k}{2}\right)-T g\left(T, t_{1}\right)\left(a+\frac{b T}{2}+\frac{c T^{2}}{3}+\right.\right. \\
& \left.\frac{d T^{3}}{4}-K\right)
\end{aligned}
$$




$$
\begin{aligned}
& \left.+\frac{\left(g\left(T, t_{1}\right)\right)^{2}}{2}\left(a+\frac{b g\left(T, t_{1}\right)}{3}+\frac{c\left(g\left(T, t_{1}\right)\right)^{2}}{6}+\frac{d\left(g\left(T, t_{1}\right)\right)^{3}}{10}-K\right)\right] \\
& +C_{L} a\left(T-g\left(T, t_{1}\right)\right)+\frac{b}{2}\left(T^{2}-\left(g\left(T, t_{1}\right)\right)^{2}\right)+\frac{c}{3}\left(T^{3}-\left(g\left(T, t_{1}\right)\right)^{3}\right)+\frac{d}{4}\left(T^{4}-\left(g\left(T, t_{1}\right)\right)^{4}\right)+\frac{1}{6 \delta^{4}}\left[6 \left\{b \delta^{3}+\right.\right. \\
& \left.c \delta^{2}(1+\delta T)+d \delta \quad(1+\delta T)^{2}\right\}\left(T-g\left(T, t_{1}\right)\right)+3\left\{c \delta^{3}+d \delta^{4}(1+\delta T) \quad\right\}\{1-\quad[1+\delta(T- \\
& \left.\left.\left.g\left(T, t_{1}\right)\right)\right]^{2}\right\}+2 d\left\{1-\left[1+\delta\left(T-g\left(T, t_{1}\right)\right)\right]^{3}\right\}-6\left\{a \delta^{3}+b \delta^{2}(1+\delta T)+\quad c \delta(1+\delta T)^{2}+d(1+\right. \\
& \left.\delta T)^{3}\right\} \log \left[\left[1+\delta\left(T-g\left(T, t_{1}\right)\right)\right]\right]
\end{aligned}
$$

\section{Approximation Solution Procedure}

To minimize total average cost per unit time, the optimal values of $t_{1}$ and $T$ can be obtained by solving the following equations simultaneously.

Provided, they satisfy the following conditions.

$$
\begin{aligned}
\frac{\partial C}{\partial t_{1}} & =0 \\
\frac{\partial C}{\partial T} & =0 \\
\text { and } \quad & \frac{\partial C}{\partial K}=0
\end{aligned}
$$

$$
\frac{\partial^{2} C}{\partial t_{1}^{2}}>0, \quad \frac{\partial^{2} C}{\partial T^{2}}>0 \quad \text { and } \quad \frac{\partial^{2} C}{\partial K^{2}}>0
$$

The numerical solution of equations (25), (26) and (27) can be obtained by using suitable computational numerical method.

\section{Conclusion}

In the present paper a volume flexible production inventory model is developed for deteriorating items with time dependent demand rate. The demand rate is taken as cubic function of time and production rate is decision variable. Production cost becomes a function of production rate. Unit production cost is depending upon material cost, Labor cost and tool or die cost. The deteriorating of unit in an inventory system is taken to weibull distribution. Shortage with partially backlogged are allowed a very natural phenomenon in inventory model. Many researchers have considered a constant backlogging rate but in many real situations, such as fashionable commodities and medicines etc., the length of the waiting time for the next replenishment would be accepted. So backlogging rate is considered to be a waiting time for next replenishment. In this model, we considered total average cost and solution procedure are also discussed.

\section{References}

[1]. Abad, P.L. (1996): Optimal pricing and lot sizing under conditions of perishabilityand partial backlogging. Management Science, 42(8), 1093-1104.

[2]. Aggarwal, S.P. and Jain, V. (2001): Optimal inventory management for exponentially increasing demand with deterioration. International Journal of Management and Systems, 17(1), 1-10.

[3]. Balkhi, Z.T. and Benkherouf, L. (1996): A production lot size inventory model for deteriorating items and arbitrary production and demand rate. European Journal of Operational Research, 92, 302-309.

[4]. Chang, H.J. and Dye, C.Y. (1999): An EOQ model for deteriorating items with time varying demand and partial backlogging. Journal of the Operational Research Society, 50(11), 1176-1182.

[5]. Chung, K.T. (2000): The inventory replenishment policy for deteriorating items under permissible delay in payments. Opsearch, 37(4), 267-281.

[6]. Chu, P. and Chung, K.J. (2004): The sensitivity of the inventory model with partial backorders. European Journal of Operational Research, 152, 289-295.

[7]. Gallego,G(1993):Reduced Production rate in the economic lot scheduling problem, International Journal of Production Research,316,1035-1046.

[8]. Giri, B.C. and Chaudhuri, K.S. (1998): Deterministic models of perishable inventory with stock dependent demand rate and nonlinear holding cost. European Journal of Operational Research, 105, 467-474.

[9]. Giri, B.C. and Yun, W.Y. (2005): Optimal lot sizing for an unreliable production system under partial backlogging and at most two failures in a production cycle. International Journal of Production Economics, 95(2), 229-243.

[10]. Hollier, R.H. and Mak, K.L. (1983): Inventory replenishment policies for deteriorating items in a declining market. International Journal of Production Economics, 21, 813-826.

[11]. Husseini and Barien(2006): Ametohd of enhance volume flexible in JIT production control, International journal of production Economics, $104,653-665$.

[12]. Khanra, S. and Chaudhuri, K.S. (2003): A note on an order-level inventory model for a deteriorating item with time dependent quadratic demand. Computers and Operations Research, 30, 1901-1916.

[13]. Khouja,N and Mehraz,A(1994):An Economics production lot size modelwith imperfect and variable production rate, Operation Research Society,45,1405-1417.

[14]. Khouza, M(1995):The economic production lot size model under volume flexibility, Computer Operation Research, 22,515-523.

[15]. Khouja, M(2005): A production model for flexible production system and product with short selling season, Applied Mathematics and decision Science,4,213,-223.

[16]. Kumar, N. and Sharma, A.K. (2000): On deterministic production inventory model for deteriorating items with an exponential declining demand. Acta Ciencia Indica, XXVI M, No. 4, 305-310. 
[17]. Silver,E.A. (1990):Deliberately An optimal replenishment policy for deteriorating items with time varying and partial exponential type backlogging. Operations Research letters, 27(4), 175-184.

[18]. Park, K.S. (1982): Inventory models with partial backorders. International Journal of Systems Science, 13, $1313-1317$.

[19]. Ramesh and Jayakumar (1991):Measurment of manufacturing flexibility a value based approach, Operation Management ,10,446-467.

[20]. Sana,S. and Chaudhuri, K.S. (2003):On volume flexible stock dependent demand inventory model, Advanced Modeling and Optimization,5,197-210.

[21]. Sana,S. and Chaudhuri, K.S. (2004): On a volume flexible inventory model, Advanced modeling and Optimization,6,1-15.

[22]. Sana,S. and Chaudhuri, K.S. (2004): On Volume flexible production polices for a deteriorating item with time dependent demand and shortages, Advanced Modeling and Optimization,6,57-59.

[23]. Sana,S. and Chaudhuri, K.S. (2006): On volume flexible production policies in family production context ,Yugoslav Journal of Operation Research,16,357-371.

[24]. Sana,S. and et al. (2007):Animperfect production process in a vbolume flexible inventory model, International Journal o0f production Economics , 105,548-559.

[25]. Schweitzer,P.J.and Seidmann,A (1991): Optimization processing rates for flexible manufacturing systems, Management Sciences, 37,454-466

[26]. Silver,E.A. (1990):Deliberately slowing down out put in afamily production contyext ,International Journal of production Research,28,17-27.

[27]. Teng, J.T., Chang, C.T. and Goyal, S.K. (2005): Optimal pricing and ordering policy under permissible delay in payments. International Journal of Production Economics, 97, 121-129.

[28]. Wee, H.M. (1995): Joint pricing and replenishment policy for deteriorating inventory with declining market. International Journal of Production Economics, Volume 40, Issues 2-3, Pages 163-171. 